\title{
Carbon-Coated Magnetic
} Nanoparticle Dedicated to MRI/ Photoacoustic Imaging of Tumor in Living Mice

\author{
Yujing $\mathrm{Li}^{1 \dagger}$, Fei $\mathrm{Ye}^{2 \dagger}$, Shanxiang Zhang ${ }^{1+}$, Wenjun $\mathrm{Ni}^{2}$, Liewei $\mathrm{Wen}^{2 *}$ and Huan Qin ${ }^{1,3 *}$ \\ ${ }^{1}$ MOE Key Laboratory of Laser Life Science and Institute of Laser Life Science, Guangdong Provincial Key Laboratory of Laser Life \\ Science, College of Biophotonics, College of Biophotonics, South China Normal University, Guangzhou, China, ${ }^{2}$ Zhuhai Precision \\ Medical Center, Zhuhai People's Hospital, Zhuhai Hospital Affiliated with Jinan University, Zhuhai, China, ${ }^{3}$ Guangzhou Key Lab of \\ Spectral Analysis and Functional Probes, College of Biophotonics, South China Normal University, Guangzhou, China
}

OPEN ACCESS

Edited by:

Wenming Liu,

Central South University, China

Reviewed by:

Xiong Wang,

ShanghaiTech University, China

Wangting Zhou,

Xidian University, China

Yuecheng Shen,

Sun Yat-sen University, China

${ }^{*}$ Correspondence:

Liewei Wen

wenner1989@163.com

Huan Qin

qinghuan@scnu.edu.cn

${ }^{\dagger}$ These authors have contributed equally to this work and share first

authorship

Specialty section:

This article was submitted to Biomaterials,

a section of the journal Frontiers in Bioengineering and

Biotechnology

Received: 24 October 2021 Accepted: 08 November 2021 Published: 02 December 2021

Citation:

Li Y, Ye F, Zhang S, Ni W, Wen L and Qin H (2021) Carbon-Coated Magnetic Nanoparticle Dedicated to MRI/ Photoacoustic Imaging of Tumor in Living Mice.

Front. Bioeng. Biotechnol. 9:800744. doi: 10.3389/fbioe.2021.800744
Multimodality imaging can reveal complementary anatomic and functional information as they exploit different contrast mechanisms, which has broad clinical applications and promises to improve the accuracy of tumor diagnosis. Accordingly, to attain the particular goal, it is critical to exploit multimodal contrast agents. In the present work, we develop novel cobalt core/carbon shell-based nanoparticles (Cobalt at carbon NPs) with both magnetization and light absorption properties for dual-modality magnetic resonance imaging (MRI) and photoacoustic imaging (PAI). The nanoparticle consists of ferromagnetic cobalt particles coated with carbon for biocompatibility and optical absorption. In addition, the prepared Cobalt at carbon NPs are characterized by transmission electron microscope (TEM), visible-near-infrared spectra, Raman spectrum, and X-ray powder diffraction for structural analysis. Experiments verify that Cobalt at carbon NPs have been successfully constructed and the designed Cobalt at carbon NPs can be detected by both MRI and PAI in vitro and in vivo. Importantly, intravenous injection of Cobalt at carbon NPs into glioblastoma-bearing mice led to accumulation and retention of Cobalt at carbon NPs in the tumors. Using such a multifunctional probe, MRI can screen rapidly to identify potential lesion locations, whereas PAl can provide high-resolution morphological structure and quantitative information of the tumor. The Cobalt at carbon NPs are likely to become a promising candidate for dual-modality MRI/PAI of the tumor.

Keywords: carbon-coated magnetic nanoparticle, multimodality imaging, photoacoustic imaging, magnetic resonance imaging, glioblastoma

\section{INTRODUCTION}

Complete surgical resection is the primary method for most solid tumors. However, because of the aggressive growth of tumor cells, clinicians face significant challenges in identifying and completely removing cancer tissue that is scattered sporadically (Baroncini et al., 2007; Issard et al., 2021). Furthermore, residual tumor tissues after surgery probably that are triggered usually leads to cancer recurrence and adverse sequelae. For achieving more complete tumor resections, many efforts have been made to explore many techniques in tumor imaging. For instance, magnetic resonance imaging 
(MRI) has been used to guide stereotactic surgical removal of tumors for preoperative planning (He et al., 2017; Ding et al., 2019; Wang et al., 2019; Liu et al., 2019; Bucci et al., 2004). However, because of tissue displacement, the tumor boundary delineated by this method in preoperative MRI is inconsistent with the actual tumor boundary during surgery (Reinges et al., 2004); consequently, the guidance of surgical resection by preoperative localization alone is limited.

Intraoperative imaging exhibits favorable potential in guiding tumor resection by improving the ratio of target background to distinguish tumor tissues (Chi et al., 2014). In the intraoperative imaging modalities used for image-guided resection, optical imaging based on tissue intrinsic optical properties or exogenous contrast agents has the advantages of fast acquisition speed, high sensitivity, convenient operation, and affordable running cost. However, because of strong light scattering, the resolution and penetration depth of these optical imaging techniques are limited, ultimately limiting the detection and localization of tumors below the surface (Razansky et al., 2009; Niu et al., 2012; Chen et al., 2017).

Photoacoustic imaging (PAI) overcomes the limitation of light diffusion by combining photoexcitation with photoacoustic detection to realize deeper target imaging with high spatial resolution (Xu and Wang, 2006; Wang and $\mathrm{Hu}, 2012$; $\mathrm{Hu}$ et al., 2019; Kim et al., 2010). It uses laser pulses to irradiate a target sample, which absorbs the laser energy, resulting in rapid thermoelastic expansion of the absorber that excites a wide-band ultrasound wave. A back-projected reconstruction algorithm is used to reconstruct a map of the distribution of the optical energy deposition within the living in living subjects (Yin et al., 2004; Yang et al., 2005; Yang et al., 2007; Yang et al., 2007; Yang et al., 2008; Xiang et al., 2009). PAI is a non-invasive imaging method that depends on the differences in the light absorption coefficients of biological tissues. In biological tissues, PA signals vary with the distribution of light absorption. Thus, PAI resembles to optical imaging in that it has a high contrast and sensitivity to tissue features. (Huang et al., 2013; Qin et al., 2013; Zhang et al., 2014; Qin et al., 2015). However, in PAI technology, the carrier of light absorption characteristics is not light signal but ultrasonic signal, and the penetration of sound to biological tissues is several orders of magnitude higher than that of light. As a result, PAI offers a far greater depth of penetration and scalable spatial resolution than other optical imaging methods (Razansky et al., 2009; Wang and Yao, 2016; Wang, 2009; Liang et al., 2021; Cheng et al., 2020).

Distinguishing tumors, especially tumors at the early stage, is extremely difficult using an imaging system only. Accurate localization and resection of tumor by imaging probe is very important for improving surgical prognosis. In particular, with the help of multimodal imaging probes, it is able to provide more comprehensive image of tumors benefiting from the contrast improvement (Lv et al., 2020). The development of a dualchannel MRI and PAI probe can be isolated by the tumor and retained long enough, so that a single injection of this drug will aid in intraoperative resection and preoperative planning of the tumor, even subtissue tumors. (Liao et al., 2019). Recently, cobaltbased nanoparticles have being extensively investigated for MRI and PAI contrast agents, owing to great optical absorption properity, magnetocrystalline anisotropy, and large magnetostrictive coefficient. Nevertheless, it is still a bottleneck problem to improve the biocompatibility of cobalt-based nanoprobes and the efficacy of dual-modality imaging to be more conducive to their clinical transformation.

Here, we have developed novel cobalt core/carbon shell-based nanoparticles (Cobalt at carbon NPs) for dual-modality MRI and PAI. To improve biocompatibility and absorption of light, nanomaterials are composed of cobalt particles coated with carbon (Figure 1A). The designed Cobalt at carbon NPs can be detected by both MRI and PAI in vitro or in vivo. After intravenous injection of cobalt at carbon nanotubes into mice carrying glioblastoma, the enhanced permeation and retention (EPR) effect caused tumor accumulation and retention of Cobalt at carbon nanotubes, allowing the use of two-channel noninvasive tumor detection. With Cobalt at carbon NPs, there is great potential to develop multiscale complementary imaging schemes. MRI can identify the location of tumor for preoperative planning, and high spatial resolution PAI provides subsequent precise blood vessel morphology and tumor imaging from the surface to the depths to accurately guiding tumor resection (Figure 1B).

\section{RESULTS}

\section{Synthesis and Characterization of Cobalt at Carbon NPs}

Cobalt nanoparticles cannot be used as MRI contrast agents on account of their oxidation-induced instability and toxicity. However, carbon coating has good biocompatibility and good air isolation, which can overcome the above shortcomings of cobalt nanoparticles. Furthermore, carbon enables optical absorption that can be used in PAI. To analyze the structure of Cobalt at carbon NPs, a series of verification experiments were performed. Transmission electron microscope (TEM) revealed that the average size of the Cobalt at carbon NPs was less than $50 \mathrm{~nm}$ (Figure 2A). The thickness of the carbon shell is about $3 \mathrm{~nm}$ (insert in Figure 2A). UV/visible-near-infrared spectra of the Cobalt at carbon NPs with different concentrations in solution $(6.25,12.5,25$, and $50 \mu \mathrm{g} / \mathrm{ml})$ shown that Cobalt at carbon NPs exhibit optical response (Figure 2B), making it amenable to PAI studies. A Raman spectrum (excitation $1,064 \mathrm{~nm}$ ) of the Cobalt at carbon NPs show the G and D bands of carbon (Figure 2C), providing evidence for the graphitic shell (Tuinstra and Koenig, 1970). We identified a crystalline body-centered-cubic Cobalt at carbon core for nanocrystals by powder X-ray diffraction (XRD, Figure 2D). As shown in powder-XRD data for nanocrystals (Figure 2D). The small peaks marked with an asterisk on the XRD curve of the nanocrystals correspond to face-centered-cubic Cobalt. These experiments demonstrated that Cobalt at carbon NPs have been successfully constructed.

\section{PAI and MRI of Cobalt at Carbon NPs in vitro}

We further validated that Cobalt at carbon NPs can enhance both PAI and MRI contrast. $T_{2}$-weighted spin echo image of Cobalt at 

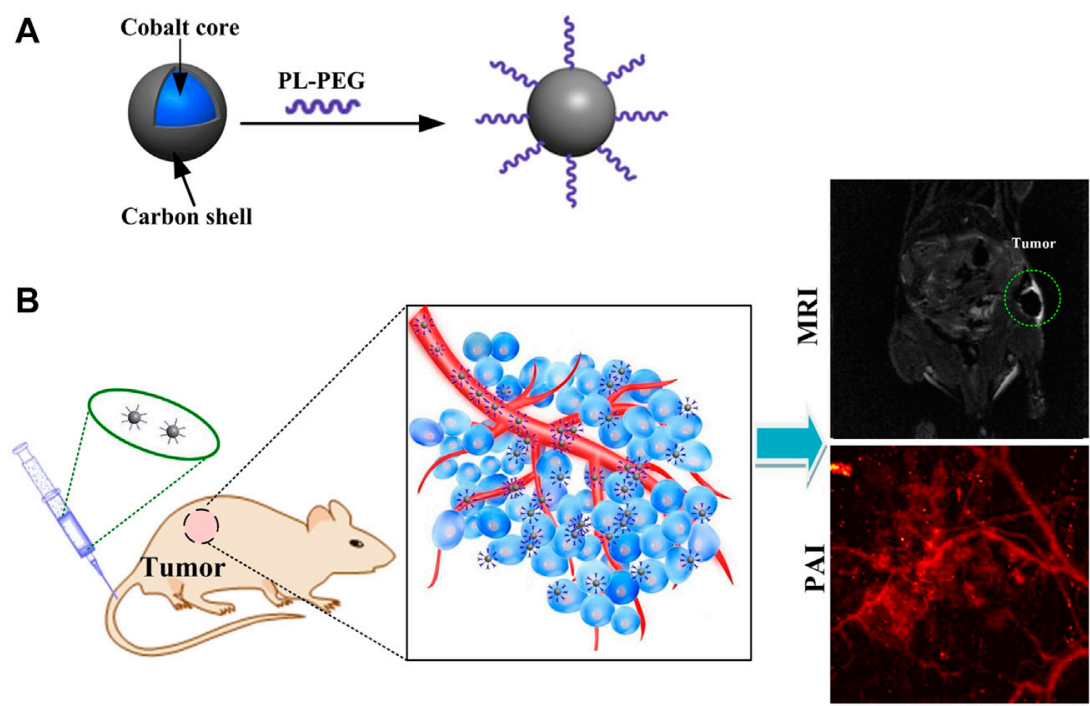

FIGURE 1 | (A) Schematic diagram of the surface modification and preparation of the cobalt core/carbon shell nanoparticles (Cobalt at carbon NPs). (B) Schematic illustration of tumor-targeted dual-modality MR/photoacoustic imaging of Cobalt at carbon NPs.

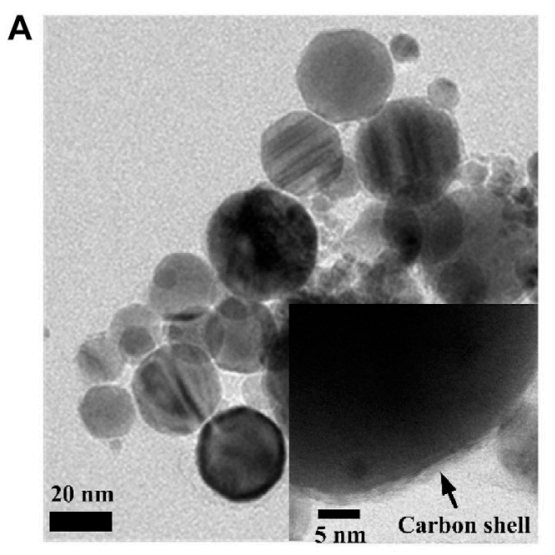

C

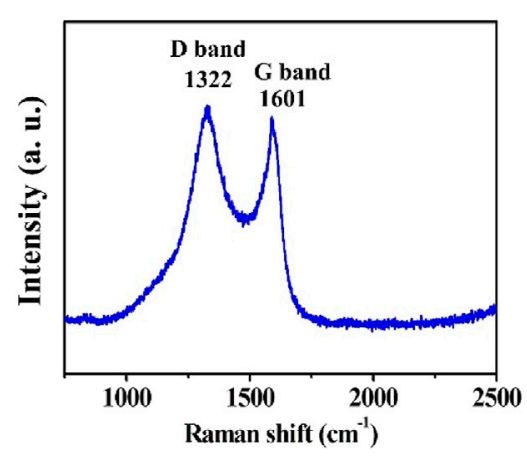

B

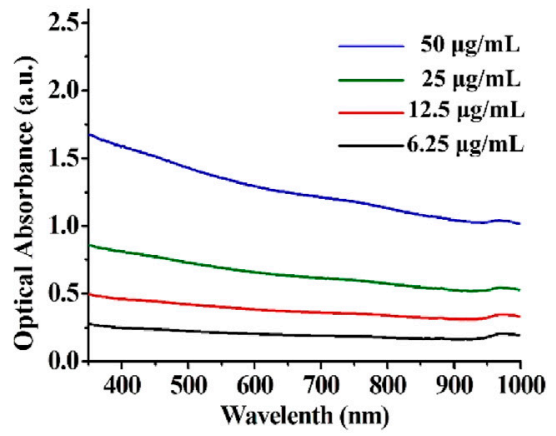

D

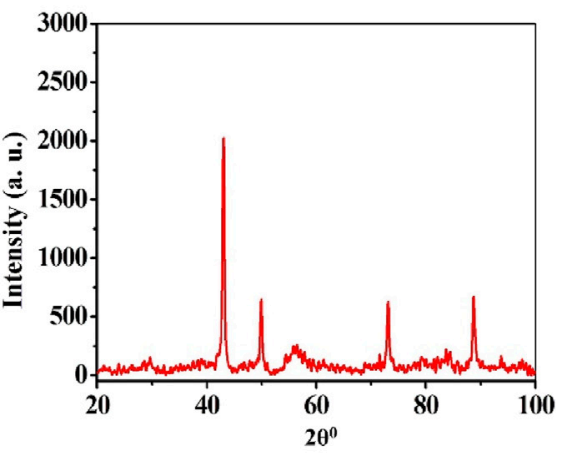

FIGURE 2 | Structural analysis of the Cobalt at carbon NPs. (A) Transmission electron microscope (TEM) images of the Cobalt at carbon NPs. (B) UV/visible-nearinfrared spectra of the Cobalt at carbon NPs with different concentrations in solution (6.25, 12.5, 25, and $50 \mathrm{\mu g} / \mathrm{ml})$. (C) Raman spectrum (excitation 1,064 nm) of the Cobalt at carbon NPs, showing the G and D bands of carbon. (D) Powder XRD data for nanocrystals. 


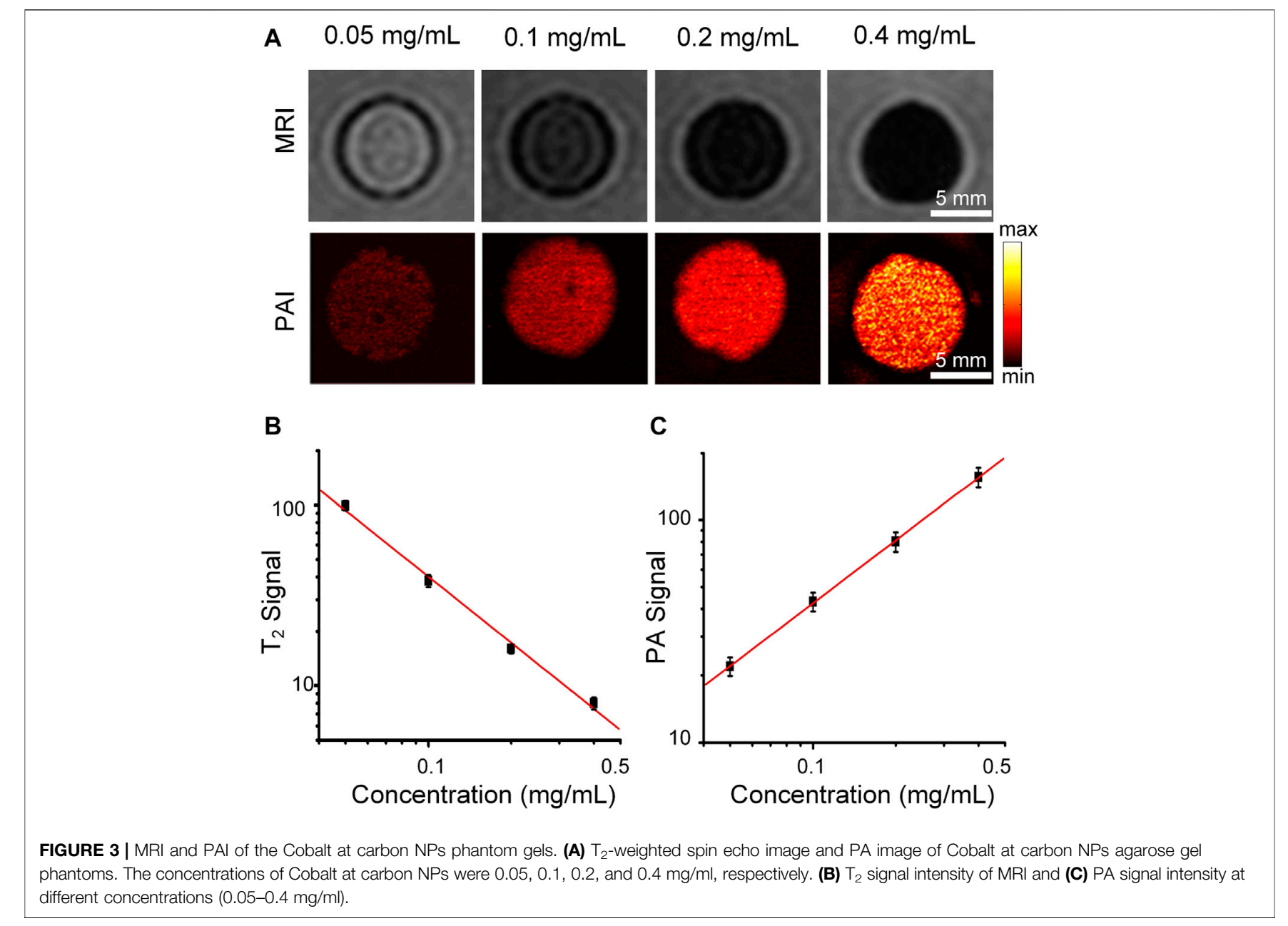

carbon NPs agarose gel phantoms are shown in Figure 3A. $\mathrm{T}_{2^{-}}$ weighted spin echo images exhibit that higher concentrations bring about lower MRI signals, indicating shorter $T_{2}$ values for water protons (Figure 3A). As the concentration of Cobalt at carbon NPs increased, MR signal intensity decreased (Figure 3B). The significant change in the MRI signal intensity can be attributed to ferromagnetic cobalt in the core of the nanoparticle. For the PAI test, some absorption-free and scatter-free agarose membranes containing cobalt at carbon nanotubes with concentrations increased from $0.05,0.1,0.2$, and $0.4 \mathrm{mg} / \mathrm{ml}$ were constructed $(\mathrm{n}=3$ inclusions of each concentration). The PA signals generated by Cobalt at Carbon NPs were highly correlated with the concentration of nanoparticles $\left(R^{2}=0.980\right.$, Figure $\left.3 \mathrm{C}\right)$. In addition, PA imaging produced by gel blocks containing Cobalt at Carbon NPs has a high level of edge differentiation, indicating its potential to identify samples or tumor edges. These experiments demonstrated that the Cobalt at carbon NPs could be used for improving both $\mathrm{MR}$ and photoacoustic signals and have good MRI and PAI dual-mode imaging ability.

The cellular uptake, and cells PAI of Cobalt at carbon NPs

To test whether Cobalt at carbon NPs can be taken up by U87 cells, first, the cell cytotoxicity of Cobalt at carbon NPs was measured using a standard CCK-8 assay. U87-MG glioma cells were used as experimental cell model. Primally, U87 glioma cells were co-incubated in cell culture medium with different concentrations $(0,5,50,100,200$, and $500 \mu \mathrm{g} / \mathrm{ml})$ of Cobalt at carbon nanotubes for $24 \mathrm{~h}$. The Cobalt at carbon NPs had no obvious toxic effect that affected the cytoactive of the U87-MG glioma cells (Figure 4A). Then, the subcellular localization of Cobalt at carbon NPs-Cy5.5 was investigated in cultured tumor cells. Nanoparticles were used to incubate U87-MG cells, whereas PBS was used to incubate U87 cells as a control, and the same treatment was then applied to all groups. Under confocal microscopy, fluorescence signals were detected on the cell membranes of Cobalt at carbon NPs-Cy5.5-incubated U87 cells (Figure 4C), whereas very small signals were detected in U87-MG cells incubated with PBS. Flow cytometry was used to further study the cell uptake characteristics. The cell uptake of Cobalt at carbon NPs-Cy5.5 was higher than that of PBS group (Figure 4B). These above results suggested that Cobalt at carbon NPs could be taken up by U87-MG cells.

To further prove that Cobalt at Carbon NPs can be taken up by U87-MG cells and have PAI ability on U87-MG cells, U87-MG cells were cultured with Cobalt at Carbon NPs $(100 \mu \mathrm{g} / \mathrm{ml})$ for $4 \mathrm{~h}$, and PAI was performed as the control group. PAI was also 


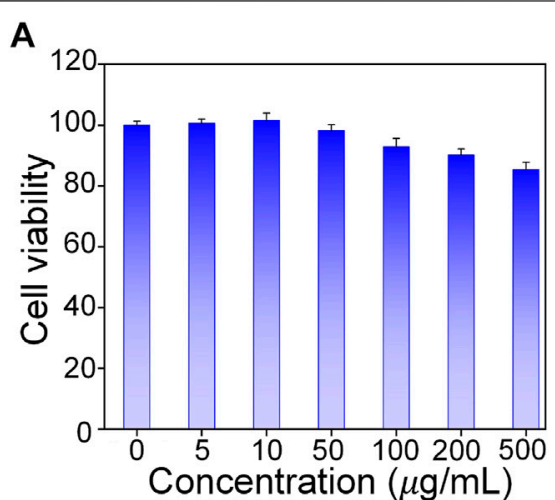

c Fluorescence
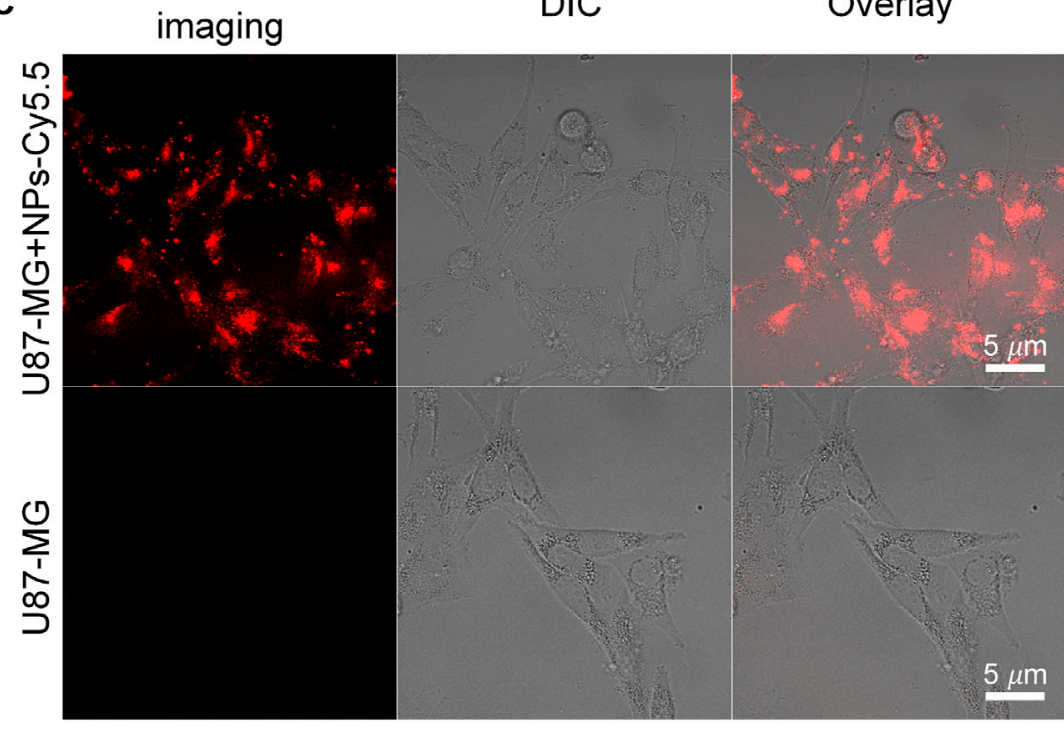

D

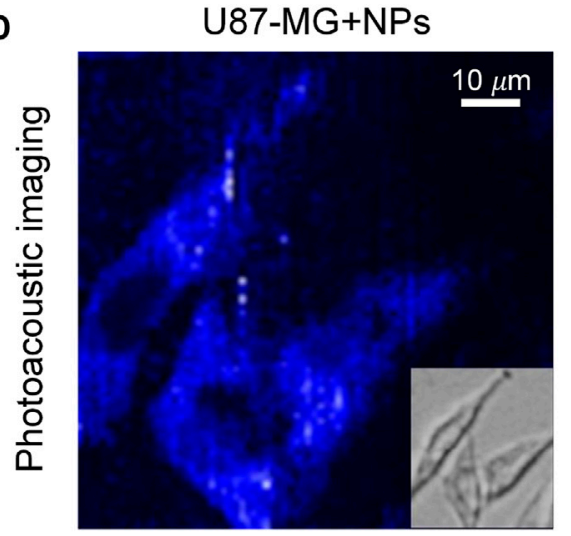

B

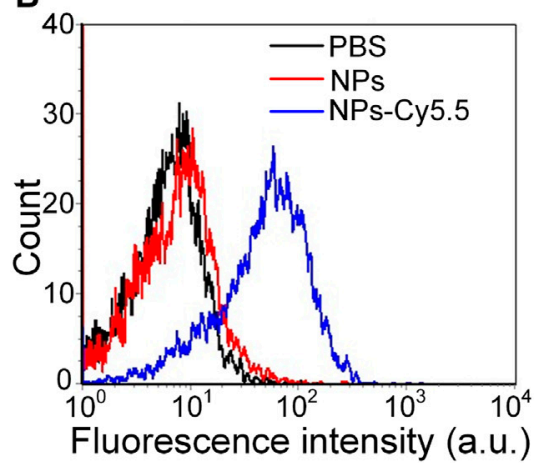

DIC

Overlay

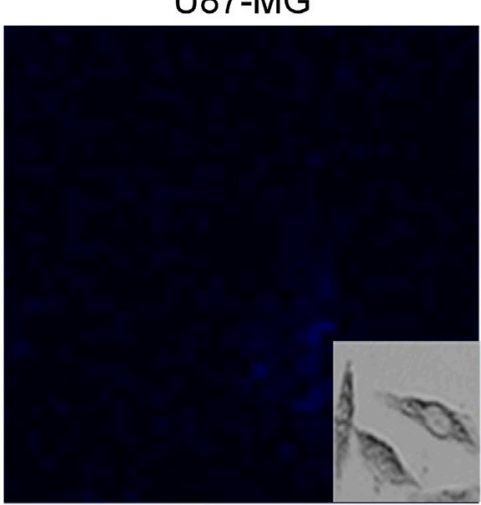

FIGURE 4 | Experiments to test the targeting ability of Cobalt at carbon NPs in vitro. (A) After culture with various concentrations of Cobalt at carbon NPs for 24 h. (B) Flow cytometry analysis of U87-MG cells incubated with PBS, Cobalt at carbon NPs, and Cobalt at carbon NPs-Cy5.5. (C) Cell uptake of Cobalt at carbon NPs-Cy5.5 in U87-MG cells. The fluorescence images were taken by confocal microscopy. (D) In vitro PAl of U87-MG cells after $4 \mathrm{~h}$ treatment with Cobalt at carbon NPs.

performed on U87-MG cells cultured without Cobalt at carbon NPs. Figure 4D shows that U87-MG cells cultured with Cobalt at carbon NPs can be fully displayed by PAI, whereas cells in the control group cannot be observed with PAI. This further indicates that Cobalt at carbon NPs can be absorbed by U87-MG cells and have excellent PIA ability.

\section{MRI and PAI of Tumor in vivo}

Finally, the advantage of combined MRI and PAI for tumor imaging in vivo is demonstrated. We attempted to test whether the Cobalt at carbon NPs could be used for tumor detection in living mice. Mice bearing U87 tumors ( $\mathrm{n}=3$ mice) were used for both MRI. After validating that Cobalt at carbon NPs had no 

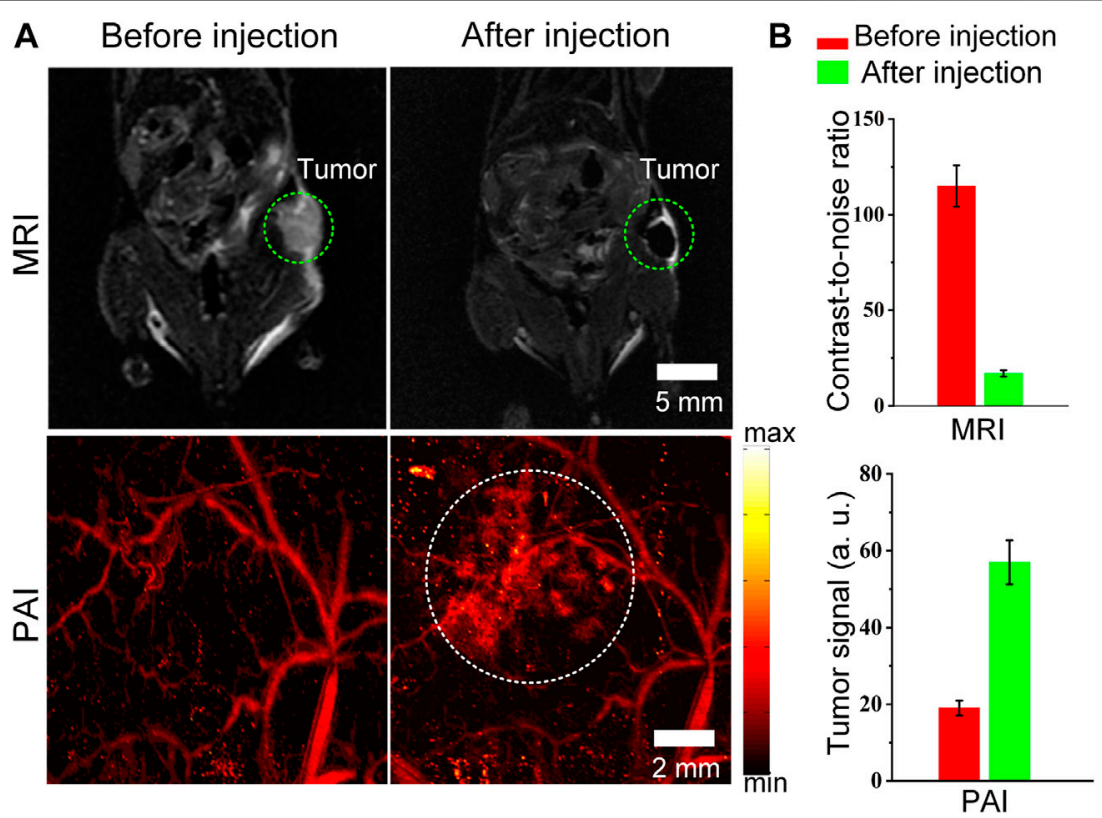

FIGURE 5 |MRI and PAI detection of tumors in living mice with Cobalt at carbon NPs (A) Two-dimensional axial MRI and photoacoustic images. The post-injection images of both modalities showed clear tumor visualization (B) Quantification of the signals in the tumor showing a significant change in the MRI and PA signals after as compared to before the injection ( $n=3$ mice).

apparent toxicity at low concentration $(1 \mathrm{mg} / \mathrm{ml})$ in vitro (data not shown). The $200 \mu \mathrm{l}$ of Cobalt at carbon NPs $(2 \mathrm{mg} / \mathrm{ml})$ were injected into mice by tail vein. Both MR and photoacoustic images of tumors before and after injection of Cobalt at carbon NPs were acquired. The low signal regions in the $\mathrm{T}_{2}$-weighted MR images indicate the locations of accumulated Cobalt at carbon NPs (Figure 5A). The nanoparticles accumulate within the tumor without necessitating a targeting mechanism that could be contributed to EPR effect, as new tumor vessels that sprout from existing vessels are often leaky, with large pores. Therefore, a high-resolution photoacoustic image of tumor was obtained. In the PA image of the tumor, the deformity of the vascular morphology was clearly shown (Figure 5A). At $6 \mathrm{~h}$ after injection of Cobalt at carbon NPs, the PA signal around the blood vessels increased, which indicated that the Cobalt at carbon NPs permeating from the blood vessels and are retention in the tumors (Figure 5A). The quantification of tumor signals in areas of interest showed significant changes in both MRI and PAI compared to pre-caudal vein injection (Figure 5B). The MRI contrast-to-noise ratio decreased from $115 \pm 10.7$ to $18 \pm 1.65 \mathrm{AU}$. The PA signal improved by $\sim 96 \%$, from $29 \pm 2.95$ to $57 \pm 5.8$ AU. The results demonstrate that Cobalt at carbon NPs could accumulate and detain in the tumors, allowing for multiscale tumor imaging using MRI and PAI. With Cobalt at carbon NPs, a multi-scale complementary imaging protocol can be established: MRI can discern the location of tumor for preoperative planning, and high spatial resolution PAI provides subsequent precise blood vessel morphology and tumor imaging from the surface to the depths to accurately guide tumor resection.

\section{In vivo Photothermal Therapy Potential}

Carbon nanomaterials have good light absorption properties in the visible wavelength range. Therefore, Cobalt at carbon NPs can achieve high light absorption and have the potential to perform photothermal therapy (PTT) on tumors. To assess the potential of Cobalt at carbon NPs to achieve PTT against tumors in living mice, we established U87-MG tumors on the back of Balb/C nude mice. After the dorsal tumor volume reached $80 \mathrm{~mm}^{3}$, eight tumor-bearing mice were randomly divided into two groups and received the same amount of $(100 \mu \mathrm{l})$ PBS and Cobalt at carbon NPs $(1 \mathrm{mg} / \mathrm{ml})$ via tail vein. After $6 \mathrm{~h}$ of injection, the tumor area was irradiated with an $808-\mathrm{nm}$ laser $\left(1 \mathrm{~W} / \mathrm{cm}^{2}\right)$ for $5 \mathrm{~min}$. At the same time, these mice were thermally imaged with an infrared thermal imager, and temperature changes were recorded (Figure 6). Under 808-nm laser irradiation, tumor temperature of mice injected with PBS only increased $9.2^{\circ} \mathrm{C}$. In contrast, the tumor site temperature of mice injected with Cobalt at carbon NPs increased by $16.3^{\circ} \mathrm{C}$ after $808-\mathrm{nm}$ laser irradiation for $5 \mathrm{~min}$. At this time, the maximum temperature reached $48.8^{\circ} \mathrm{C}$, which was sufficient to achieve photothermal ablation of the tumor (Lu et al., 2005; Day et al., 2012; MacDonald et al., 2014; Li et al., 2016; Shao et al., 2016). These results suggest that Cobalt at carbon NPs have the possibility of realizing PTT in vivo mouse tumors.

\section{DISCUSSION}

MRI, providing both anatomic and functional information, is one of the most powerful noninvasive imaging methods to image the whole human body in clinical diagnosis and prognosis. PAI is a 


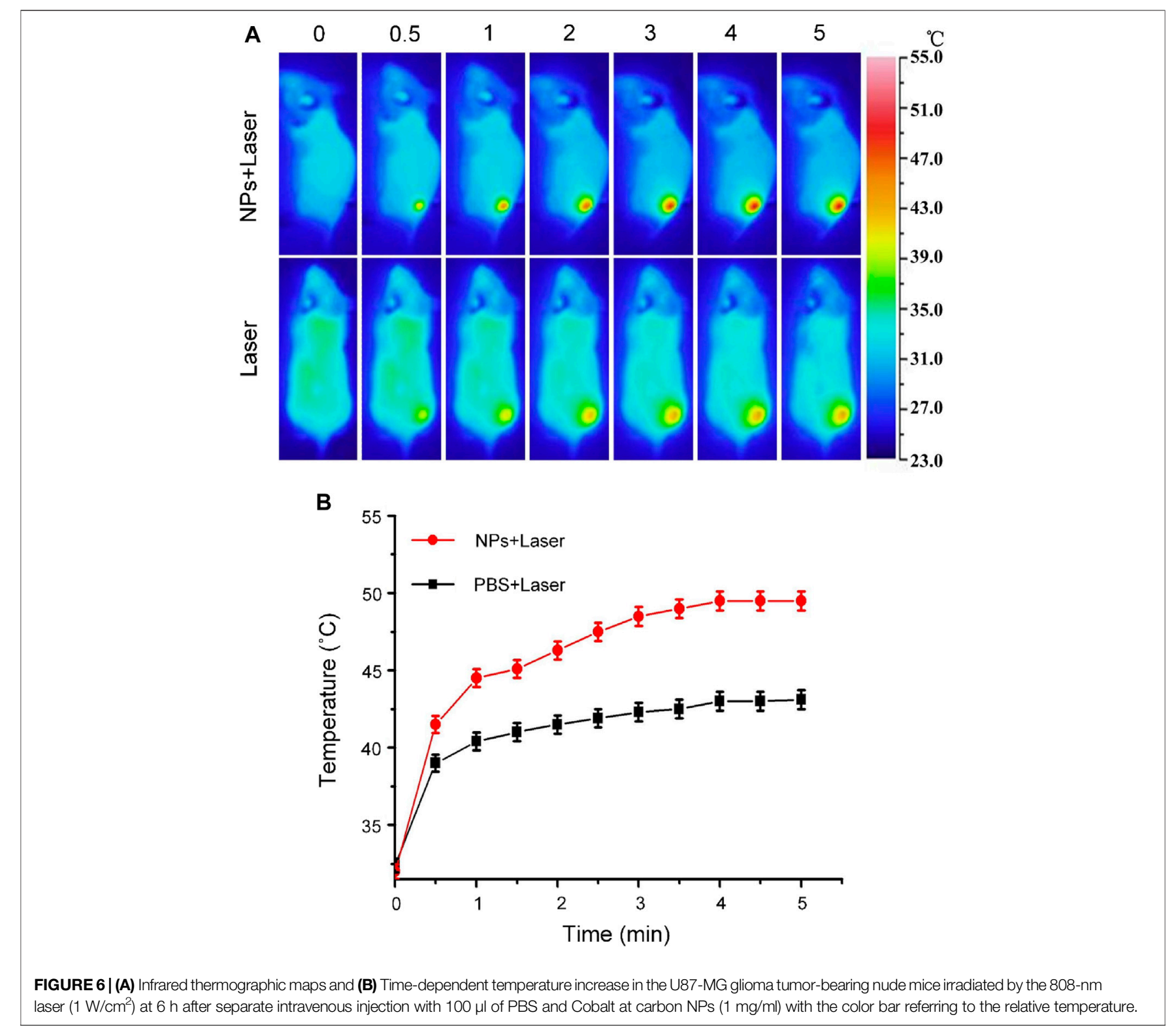

new technique that depends on the contrast generated by the optical absorption property of the tissues. Compared with traditional optical imaging, PA imaging shows remarkably improved imaging depth, owing to the excellent soft tissue penetration ability of sound, and is capable to achieve high resolution imaging in deep-seated tissue successfully. Cobalt at carbon NPs with magnetization and optical absorption properties may thus be used as contrast agents for PAI and MRI contrasts simultaneously. When Cobalt at carbon NPs can orientation to the tumor site, preoperative and intraoperative imaging of tumor could be realized. Although several kinds of dual-modality PAI and MRI probes have been developed (Kircher et al., 2012; Bouchard et al., 2009), carbon shell-based nanoparticles (Cobalt at carbon NPs) with excellent biocompatibility are candidates for PAI and MRI dual-mode imaging. In addition, Cobalt at carbon NPs show good photothermal effect and have the potential of PTT for tumors. Herein, Cobalt at carbon NPs are concentrated at the tumor site through EPR effect. However, it has been reported that targeting molecules [such as RGD (Biscaglia et al., 2019; Liang et al., 2021; Cao et al., 2020) and folic acid (Prossnitz and Barton, 2014; De Marco et al., 2016)] that can specifically recognize tumor cells through surface connection can enhance the aggregation of nanoparticles at the tumor site and improve imaging and PTT ability. Hence, Cobalt at carbon NPs can probably be a candidate multifunctional nanodrug for dual-mode image-guided tumor PTT in the future. Detailed pharmacokinetic, in vivo toxicity, and PTT studies will be carried out in the next step.

In addition, to promote the clinical application of Cobalt at Carbon NPs, further exploration can be made from the following aspects: 1) To promote the clinical application of Cobalt at carbon NPs, first of all, it is necessary to ensure that their 
biocompatibility and biosafety are high enough, and further verification of the biocompatibility of new Cobalt at carbon NPs in organisms is required. 2) Add targeted molecules to improve the targeting ability, so that nanoparticles can specifically gather to the tumor site. 3) Studies have shown that the larger the size of the nanoprobe, the higher its uptake by the liver and the smaller the amount of the nanoprobe reaching the tumor site. High uptake of contrast agents by tumors can be achieved by reducing the size of nanoparticles.

\section{CONCLUSION}

In summary, Cobalt at carbon NPs were successfully developed for the first time as a novel probe for MRI and PAI. The nanoprobes were obtained by coating carbon onto the cobalt nanoparticle. Cobalt at carbon NPs possess both ferromagnetic and optical absorption properties, which could be used for enhancing both MRI and photoacoustic signals both in vitro and in vivo. After intravenous injection of Cobalt at carbon NPs, Cobalt at carbon NPs can accumulate and retain in tumors, allowing for a noninvasive tumor imaging using by MRI and PAI system. With Cobalt at carbon NPs, it is possible to institute a multiscale complementary imaging protocol that MRI can screen to identify the location of tumor for preoperative planning, and high spatial resolution PAI provides subsequent precise blood vessel morphology and tumor imaging from the surface to the depths to accurately guide tumor resection.

\section{METHODS AND MATERIALS}

\section{Materials}

Cobalt at carbon NPs were purchased from Sigma-Aldrich Co. Ltd. (Shanghai, China). Phospholipid-poly(ethylene glycol) (PL-PEG, $\mathrm{MW}$ of PEG $=5,000$ ) were purchased from JenKem Technology Co. Ltd. Fluorescein isothiocyanate-annexin V, propyl iodide, and calcein acetoxymethyl ester were obtained from Kumamoto Dojindo Laboratories, Japan. All chemicals purchased are analytical grade and can be used directly without further purification. All the water used in the experiment is high-purity deionized water (resistance $>18 \mathrm{M} \Omega \mathrm{cm}$ ). Animal handling procedures and animal care and Use are in full compliance with the relevant guidelines of the Animal Care and Use Council.

\section{PL-PEG-Functionalized Cobalt at Carbon NPs}

Through the non-covalent functionalization of phospholipidpolyethylene glycol, a stable aqueous suspension of Cobalt at carbon nanotubes was prepared. The hydrocarbon chains of the phospholipids are adsorbed to the graphite shell through van der Waals and hydrophobic interactions, whereas the hydrophilic PEG chains extend to the water phase to achieve dissolution. Add PL-PEG-functionalized Cobalt at carbon nanotubes to the newly synthesized Cobalt at carbon nanotube solution $(2 \mathrm{ml}, 2 \mathrm{mg} / \mathrm{ml})$, stir for $12 \mathrm{~h}$ to ensure that the polymer coating is complete. Then, centrifuging $(8,000 \mathrm{rpm}, 20 \mathrm{~min})$, eliminate unbound PL-PEG molecules and prepare PL-PEG-functionalized cobalt at carbon nanotubes.

\section{Characterization Experiments}

A HITACHI H-300 transmission electron microscope (TEM) was used to observe the samples with the parameters of $70-\mathrm{kV}$ voltage and 70-pA current. Raman spectroscopy was used to detect the $G$ and $D$ bands of carbon. Use 1,064-nm laser excitation. After focusing at the center of the capillary, record the Raman spectrum of the sample. X-ray diffractometer (Bruker diffractometer, $3-\mathrm{kW} \mathrm{Cu}-\mathrm{Ka}$ radiation) was used to analyze the Cobalt core/carbon shell nanocrystals.

\section{Magnetic Resonance Imaging in vitro}

The Cobalt at carbon NPs were diluted with deionized water (18.2 $\mathrm{M} \Omega$ resistivity) to prepare Cobalt at carbon NPs with different concentrations. The diluted core/carbon NPs sample is in an agar phantom. MRI uses 1.5-T imager (GE Signa HD, 1.5 T MR, GE Healthcare, Milwaukee, WI, United States) and $12.7 \mathrm{~cm}$ only accepts knee joint coils. The magnetic resonance coronal image is scanned with a fast spin echo $\mathrm{T}_{2}$ sequence [repetition time $(\mathrm{ms}) /$ echo time $(\mathrm{ms})=4,000 / 108$, echo sequence length is 16], and an image with a matrix size of $256 \times 256$ is obtained.

\section{Photoacoustic Imaging System}

A miniature laser (Laser-export Co. Ltd., Moscow, Russia), with a working wavelength of $532 \mathrm{~nm}$, a pulse width of $<10 \mathrm{~ns}$, and a repetition frequency of up to $30 \mathrm{kHz}$, is used as the excitation light source for the PAI system. After the light passes through the scanning lens and the tube lens, it is focused by the objective lens and illuminates the sample under test. The average energy density of the laser was set to $<20 \mathrm{~mJ} \mathrm{~cm}{ }^{-2}$. After that, the PA signal generated by the tested sample is received by an ultrasonic transducer with a center frequency of $10 \mathrm{MHz}$ and a bandwidth of $-6 \mathrm{~dB}$ (China Doppler Electronic Technology Co., Ltd.). The photoacoustic signal is recorded by the computer through the data acquisition card. The data acquisition card has a sampling rate of $200 \mathrm{M}$ samples/s. The two-dimensional scanning table is driven by a computer-controlled ultrasonic motor. The computer controls the ultrasonic motor to move the sample and analyzes the photoacoustic signal generated by each scanning point. Then, the PA image in the region of interest can be obtained.

\section{Photoacoustic Imaging in vitro}

The synthesized nanoparticles were diluted with deionized water (18.2 $\mathrm{M} \Omega$ resistivity) to prepare Cobalt at carbon NPs with different concentrations. The diluted core/carbon shell nanoparticle sample is embedded in an agar phantom. PAI is obtained through a PAI system.

\section{Cell Viability Assays}

The cell cytotoxicity of Cobalt at carbon NPs was tested using the colorimetric tetrazolium base to detect CCK-8. U87-MG cells were seeded in a 96-well microtiter plate $\left(5 \times 10^{3}\right.$ per well, $\left.100 \mu \mathrm{l}\right)$. The solutions of samples at $0 \mu \mathrm{g} / \mathrm{ml}$ (PBS), $5 \mu \mathrm{g} / \mathrm{ml}, 10 \mu \mathrm{g} / \mathrm{ml}, 50 \mu \mathrm{g} / \mathrm{ml}$, 
$100 \mu \mathrm{g} / \mathrm{ml} 200 \mu \mathrm{g} / \mathrm{ml}$, and $500 \mu \mathrm{g} / \mathrm{ml}$ were incubated for $24 \mathrm{~h}$. After that, the cell viability was tested according to the CCK-8 assay. Each experiment was conducted in five independent parallel groups.

\section{In vitro Cell Uptake}

For in vitro cell uptake test, U87 cells were seeded into a confocal cell culture dish $\left(1 \times 10^{5}\right.$ cells/well $)$ for $24 \mathrm{~h}$, and then, the initial medium was replaced with a new serum-free medium, including Cobalt at carbon NPs-Cy5. Five and PBS, incubate for $3 \mathrm{~h}$, wash with PBS three times, and observe the morphology with a confocal microscope (ZEIESS LSM SH120, Germany). Then, the samples (containing 10,000 cells) were run on a FACSCanto II flow cytometer (Becton Dickinson, Mountain View, CA, United States).

\section{Animal Culture}

Male BALB/c nude mice were purchased from the Provincial Animal Center (Guangzhou, China), 3-4 weeks old, under the standard breeding conditions.

\section{Animal Models}

The PBS dispersion of U87 MG cells $\left(1 \times 10^{6}, 100 \mu \mathrm{l}\right)$ was injected into the back area of Balb/c mice. After 1 week, the tumor grew to about $80 \mathrm{~mm}^{3}$ in size, thus successfully constructing a typical tumor transplantation model on the back of the tumor.

\section{MRI and PAl in vivo}

Glioma-bearing mice (tumor volume was $80 \mathrm{~mm}^{3}$ ) were recorded images of the T2-weighted imaging and PAI $(\lambda=532 \mathrm{~nm}$, pulse duration $=10 \mathrm{~ns}$, and pulse energy $=10 \mathrm{~mJ} \mathrm{~cm}^{-2}$ ). Then, the mice were injected with $100 \mu \mathrm{l}$ of Cobalt at carbon NPs solution

\section{REFERENCES}

Baroncini, M., Vinchon, M., Minéo, J.-F., Pichon, F., Francke, J.-P., and Dhellemmes, P. (2007). Surgical Resection of Thalamic Tumors in Children: Approaches and Clinical Results. Childs. Nerv. Syst. 23, 753-760. doi:10.1007/ s00381-007-0299-4

Biscaglia, F., Ripani, G., Rajendran, S., Benna, C., Mocellin, S., Bocchinfuso, G., et al. (2019). Gold Nanoparticle Aggregates Functionalized with Cyclic RGD Peptides for Targeting and Imaging of Colorectal Cancer Cells. ACS Appl. Nano Mater. 2, 6436-6444. doi:10.1021/acsanm.9b01392

Bouchard, L. S., Anwar, M. S., Liu, G. L., Hann, B., Xie, Z. H., Gray, J. W., et al. (2009). Picomolar Sensitivity MRI and Photoacoustic Imaging of Cobalt Nanoparticles. $P$. Nat. Acad. Sci. USA 106, 4085-4089. doi:10.1073/pnas.0813019106

Bucci, M. K., Maity, A., Janss, A. J., Belasco, J. B., Fisher, M. J., Tochner, Z. A., et al. (2004). Near Complete Surgical Resection Predicts a Favorable Outcome in Pediatric Patients with Nonbrainstem, Malignant Gliomas. Cancer 101, 817-824. doi:10.1002/cncr.20422

Cao, F., Zhou, Y., Liu, X., and Yu, C.-h. (2020). Podosome Formation Promotes Plasma Membrane Invagination and Integrin-B3 Endocytosis on a Viscous RGD-Membrane. Commun. Biol. 3, 117. doi:10.1038/s42003-020-0843-2

Chen, J., Jiang, X., Zhang, C., MacKenzie, K. R., Stossi, F., Palzkill, T., et al. (2017). Reversible Reaction-Based Fluorescent Probe for Real-Time Imaging of Glutathione Dynamics in Mitochondria. ACS Sens. 2, 1257-1261. doi:10.1021/acssensors.7b00425

Cheng, Z., Ma, H., Wang, Z., and Yang, S. (2020). In Vivo volumetric Monitoring of Revascularization of Traumatized Skin Using Extended Depth-Of-Field Photoacoustic Microscopy. Front. Optoelectron. 13 (4), 307-317. doi:10.1007/s12200-020-1040-0
$(200 \mu \mathrm{g} / \mathrm{ml})$. Six hours later, the animals were anesthetized with isoflurane, and MRI and PAI were performed.

\section{DATA AVAILABILITY STATEMENT}

The raw data supporting the conclusions of this article will be made available by the authors, without undue reservation.

\section{ETHICS STATEMENT}

The animal study was reviewed and approved by the Institutional Animal Care and Use Committee of South China Normal University (Guangzhou, China).

\section{AUTHOR CONTRIBUTIONS}

YL, FY, and SZ wrote and prepared original draft. WN, LW, and HQ reviewed and edited drafts and conceptualized this work.

\section{FUNDING}

This research was supported by the Science and Technology Planning Project of Guangdong Province, China (grant nos. 2019A1515012054; 2020A1515011101, and 2021A1515011703); and the Science and Technology Program of Guangzhou (no. 2019050001).

Chi, C., Du, Y., Ye, J., Kou, D., Qiu, J., Wang, J., et al. (2014). Intraoperative Imaging-Guided Cancer Surgery: from Current Fluorescence Molecular Imaging Methods to Future Multi-Modality Imaging Technology. Theranostics 4, 1072-1084. doi:10.7150/thno.9899

Day, E. S., Zhang, L., Thompson, P. A., Zhang, L., Thompson, P. A., Zawaski, J. A., et al. (2012). Vascular-targeted Photothermal Therapy of an Orthotopic Murine Glioma Model. Nanomedicine (Lond) 7 (8), 1133-1148. doi:10.2217/ nnm.11.189

De Marco, P., Lappano, R., De Francesco, E. M., Cirillo, F., Pupo, M., Avino, S., et al. (2016). GPER Signalling in Both Cancer-Associated Fibroblasts and Breast Cancer Cells Mediates a Feedforward IL1ß/IL1R1 Response. Sci. Rep. 6, 24354. doi:10.1038/srep24354

Ding, B., Shao, S., Jiang, F., Dang, P., Sun, C., Huang, S., et al. (2019). MnO2Disguised Upconversion Hybrid Nanocomposite: An Ideal Architecture for Tumor Microenvironment-Triggered UCL/MR Bioimaging and Enhanced Chemodynamic Therapy. Chem. Mater. 31, 2651-2660. doi:10.1021/ acs.chemmater.9b00893

He, D., Hai, L., He, X., Yang, X., and Li, H.-W. (2017). Glutathione-Activatable and O2/Mn2+ -Evolving Nanocomposite for Highly Efficient and Selective Photodynamic and Gene-Silencing Dual Therapy. Adv. Funct. Mater. 27, 1704089. doi:10.1002/adfm.201704089

Hu, Y., Chen, Z., Xiang, L., and Xing, D. (2019). Extended Depth-Of-Field AllOptical Photoacoustic Microscopy with a Dual Non-diffracting Bessel Beam. Opt. Lett. 44, 1634-1637. doi:10.1364/OL.44.001634

Huang, P., Lin, J., Li, W., Rong, P., Wang, Z., and Wang, S. (2013). Leapman.Biodegradable Gold Nanovesicles with an Ultrastrong Plasmonic Coupling Effect for Photoacoustic Imaging and Photothermal Therapy. Angew. Chem. 125, 14208-14214. doi:10.1002/anie.20130898610.1002/ anie.20130898610.1002/ange.201308986 
Issard, J., Sa Cunha, A., Fabre, D., Mitilian, D., Mussot, S., Mercier, O., et al. (2021). Surgical Resection of Tumors Invading the Inferior Vena Cava at the Hepatic Vein and Thoracic Levels. World J. Surgj. Surg. 45, 3174-3182. doi:10.1007/ s00268-021-06227-9

Kim, C., Favazza, C., and Wang, L. V. (2010). In Vivo photoacoustic Tomography of Chemicals: High-Resolution Functional and Molecular Optical Imaging at New Depths. Chem. Rev. 110, 2756-2782. doi:10.1021/cr900266s

Kircher, M. F., De La Zerda, A., Jokerst, J. V., Zavaleta, C. L., Kempen, P. J., Mittra, E., et al. (2012). A Brain Tumor Molecular Imaging Strategy Using a New Triple-Modality MRI-Photoacoustic-Raman Nanoparticle. Nat. Med. 18, 829-834. doi:10.1038/nm.2721

Lao, Y., Xing, D., Yang, S., Xiang, L., and Lao, Y. (2008). Noninvasive Photoacoustic Imaging of the Developing Vasculature during Early Tumor Growth. Phys. Med. Biol. 53, 4203-4212. doi:10.1088/0031-9155/53/15/013

Li, Z., Huang, H., Tang, S., Li, Y., Yu, X-F., Wang, H., et al. (2016). Small Gold Nanorods Laden Macrophages for Enhanced Tumor Coverage in Photothermal Therapy. Biomaterials 74, 144-154. doi:10.1016/j.biomaterials.2015.09.038

Liang, L., Wen, L., Weng, Y., Song, J., Li, H., Zhang, Y., et al. (2021). Homologoustargeted and Tumor Microenvironment-Activated Hydroxyl Radical Nanogenerator for Enhanced Chemoimmunotherapy of Non-small Cell Lung Cancer. Chem. Eng. J. 425, 1385-8947. doi:10.1016/j.cej.2021.131451

Liang, Z., Wang, Q., Liao, H., Zhao, M., Lee, J., Yang, C., et al. (2021). Artificially Engineered Antiferromagnetic Nanoprobes for Ultra-sensitive Histopathological Level Magnetic Resonance Imaging. Nat. Commun. 12, 3840. doi:10.1038/s41467-021-24055-2

Liao, J., Jia, Y., and Chen, L. (2019). Magnetic/Gold Core-Shell Hybrid Particles for Targeting and Imaging-Guided Photothermal Cancer Therapy. J. Biomed. Nanotechnol. 15 (10), 2072-2089. doi:10.1166/jbn.2019.2839

Liu, C., Wang, D., Zhang, S., Cheng, Y., Yang, F., Xing, Y., et al. (2019). Biodegradable Biomimic Copper/Manganese Silicate Nanospheres for Chemodynamic/ Photodynamic Synergistic Therapy with Simultaneous Glutathione Depletion and Hypoxia Relief. ACS Nano 13, 4267-4277. doi:10.1021/acsnano.8b09387

$\mathrm{Lu}, \mathrm{Y}$., Zhu, Z., and Liu, Z. (2005). Carbon-encapsulated Fe Nanoparticles from Detonation-Induced Pyrolysis of Ferrocene. Carbon 43, 369-374. doi:10.1016/ j.carbon.2004.09.020

Lv, K., Lin, H., and Qu, F. (2020). Biodegradable Hollow $\mathrm{Co}_{3} \mathrm{~S}_{4} @ \mathrm{~N}$-Doped Carbon as Enhanced PTT/PDT Agent for Multimodal MR/thermal Imaging and Synergistic Antitumor Therapy. Chem. Eng. J. 392, 124555. doi:10.1016/j.cej.2020.124555

MacDonald, T. D., Liu, T. W., and Zheng, G. (2014). An MRI-Sensitive, Nonphotobleachable Porphysome Photothermal Agent. Angew. Chem. Int. Ed. Engl. 53 (27), 6956-6959. doi:10.1002/anie.201400133

Niu, L.-Y., Guan, Y.-S., Chen, Y.-Z., Wu, L.-Z., Tung, C.-H., and Yang, Q.-Z. (2012). BODIPY-based Ratiometric Fluorescent Sensor for Highly Selective Detection of Glutathione over Cysteine and Homocysteine. J. Am. Chem. Soc. 134, 18928-18931. doi:10.1021/ja309079f

Prossnitz, E. R., and Barton, M. (2014). Estrogen Biology: New Insights into GPER Function and Clinical Opportunities. Mol. Cel. Endocrinol. 389, 71-83. doi:10.1016/j.mce.2014.02.002

Qin, H., Zhou, T., Yang, S., Chen, Q., and Xing, D. (2013). Gadolinium(III)-gold Nanorods for MRI and Photoacoustic Imaging Dual-Modality Detection of Macrophages in Atherosclerotic Inflammation. Nanomedicine 8, 1611-1624. doi:10.2217/nnm.12.168

Qin, H., Zhou, T., Yang, S., and Xing, D. (2015). Fluorescence Quenching Nanoprobes Dedicated to In Vivo Photoacoustic Imaging and High-Efficient Tumor Therapy in Deep-Seated Tissue. Small 11, 2675-2686. doi:10.1002/smll.201403395

Razansky, D., Distel, M., Vinegoni, C., Ma, R., Perrimon, N., Köster, R. W., et al. (2009). Multispectral Opto-Acoustic Tomography of Deep-Seated Fluorescent Proteins In Vivo. Nat. Photon 3, 412-417. doi:10.1038/nphoton.2009.98
Reinges, M. H. T., Nguyen, H.-H., Krings, T., Hutter, B.-O., Rohde, V., and Gilsbach, J. M. (2004). Course of Brain Shift during Microsurgical Resection of Supratentorial Cerebral Lesions: Limits of Conventional Neuronavigation. Acta Neurochirurgica 146, 369-377. doi:10.1007/s00701-003-0204-1

Shao, J., Xie, H., Huang, H., Li, Z., Sun, Z., Xu, Y., et al. (2016). Biodegradable Black Phosphorus-Based Nanospheres for In Vivo Photothermal Cancer Therapy. Nat. Commun. 7, 12967. doi:10.1038/ncomms 12967

Tuinstra, F., and Koenig, J. L. (1970). Raman Spectrum of Graphite. J. Chem. Phys. 53, 1126-1130. doi:10.1063/1.1674108

Wang, S.-S., Cheng, Q., Zeng, X., and Zhang, X.-Z. (2019). A Mn(III)-Sealed MetalOrganic Framework Nanosystem for Redox-Unlocked Tumor Theranostics. ACS Nano 13, 6561-6571. doi:10.1021/acsnano.9b00300

Wang, L. V., and Hu, S. (2012). Photoacoustic Tomography: In Vivo Imaging from Organelles to Organs. Science 335, 1458-1462. doi:10.1126/science.1216210

Wang, L. V. (2009). Multiscale Photoacoustic Microscopy and Computed Tomography. Nat. Photon. 3, 503-509. doi:10.1038/nphoton.2009.157

Wang, L. V., and Yao, J. (2016). A Practical Guide to Photoacoustic Tomography in the Life Sciences. Nat. Methods 13 (8), 627-638. doi:10.1038/nmeth.3925

Xiang, L., Yuan, Y., Xing, D., Ou, Z., Yang, S., and Zhou, F. (2009). Photoacoustic Molecular Imaging with Antibody-Functionalized Single-Walled Carbon Nanotubes for Early Diagnosis of Tumor. J. Biomed. Opt. 14, 021008-0210087. doi:10.1117/1.3078809

$\mathrm{Xu}, \mathrm{M}$, and Wang, L. V. (2006). Photoacoustic Imaging in Biomedicine. Rev. Scientific Instr. 77, 041101. doi:10.1063/1.2195024

Yang, D., Xing, D., Gu, H., Tan, Y., and Zeng, L. (2005). Fast Multielement PhaseControlled Photoacoustic Imaging Based on Limited-Field-Filtered BackProjection Algorithm. Appl. Phys. Lett. 87, 194101. doi:10.1063/1.2119417

Yang, S., Xing, D., Lao, Y., Yang, D., Zeng, L., Xiang, L., et al. (2007). Noninvasive Monitoring of Traumatic Brain Injury and post-traumatic Rehabilitation with Laser-Induced Photoacoustic Imaging. Appl. Phys. Lett. 90, 243902. doi:10.1063/1.2749185

Yang, S., Xing, D., Zhou, Q., Xiang, L., and Lao, Y. (2007). Functional Imaging of Cerebrovascular Activities in Small Animals Using High-Resolution Photoacoustic Tomography. Med. Phys. 34, 3294-3301. doi:10.1118/1.2757088

Yin, B., Xing, D., Wang, Y., Zeng, Y., Tan, Y., and Chen, Q. (2004). Fast Photoacoustic Imaging System Based on 320-element Linear Transducer Array. Phys. Med. Biol. 49, 1339-1346. doi:10.1088/0031-9155/49/7/019

Zhang, J., Yang, S., Ji, X., Zhou, Q., and Xing, D. (2014). Characterization of LipidRich Aortic Plaques by Intravascular Photoacoustic Tomography. J. Am. Coll. Cardiol. 64, 385-390. doi:10.1016/j.jacc.2014.04.053

Conflict of Interest: The authors declare that the research was conducted in the absence of any commercial or financial relationships that could be construed as a potential conflict of interest.

Publisher's Note: All claims expressed in this article are solely those of the authors and do not necessarily represent those of their affiliated organizations or those of the publisher, the editors, and the reviewers. Any product that may be evaluated in this article, or claim that may be made by its manufacturer, is not guaranteed or endorsed by the publisher.

Copyright $\odot 2021 \mathrm{Li}, \mathrm{Ye}$, Zhang, Ni, Wen and Qin. This is an open-access article distributed under the terms of the Creative Commons Attribution License (CC BY). The use, distribution or reproduction in other forums is permitted, provided the original author(s) and the copyright owner(s) are credited and that the original publication in this journal is cited, in accordance with accepted academic practice. No use, distribution or reproduction is permitted which does not comply with these terms. 